\title{
Movilidad ocupacional y diferencial de ingresos: la experiencia del Brasil entre 2002 y 2010
}

\author{
Sandro Eduardo Monsueto, Julimar da Silva Bichara \\ y André Moreira Cunba
}

RESUMEN

Desde comienzos del siglo XXI, la economía brasileña ha experimentado un ciclo de crecimiento de características peculiares en comparación con su experiencia histórica previa, consistentes en una combinación de crecimiento, estabilidad macroeconómica y avances en el perfil distributivo. En ese contexto, este trabajo tiene como objetivo analizar los factores y efectos distributivos de la movilidad ocupacional en el Brasil, sobre la base de los datos de la Encuesta Mensual de Empleo. Los resultados sugieren que: i) la movilidad se ha utilizado en el Brasil como vía de aumento salarial, incluso cuando da lugar a una caída del estatus socio-ocupacional; ii) sin embargo, el incremento salarial al cambiar de empleo o de segmento ocupacional es diferente para los más ricos y los más pobres y resulta menor para los segundos; iii) por consiguiente, la movilidad contribuye a acrecentar los ingresos, pero al mismo tiempo, tiende a ensanchar las diferencias salariales.

PALABRAS CLAVE

CLASIFICACIÓN JEL

AUTORES
Empleo, mercado de trabajo, movilidad de la mano de obra, salarios, igualdad, Brasil

$\mathrm{J} 31, \mathrm{~J} 62$

Sandro Eduardo Monsueto es profesor adjunto del Núcleo de Estudios e Investigaciones Económicas (Nepec) de la Facultad de Administración, Ciencias Contables y Ciencias Económicas de la Universidad Federal de Goiás, Brasil. monsueto@ufg.br

Julimar da Silva Bichara es profesor de la Universidad Autónoma de Madrid (UAM), España. julimar. dasilva@uam.es

André Moreira Cunha es profesor asociado en el Programa de Posgrado en Economía de la Universidad Federal de Rio Grande do Sul (ufrgs) e investigador del Consejo Nacional de Desarrollo Científico y Tecnológico (cNPq), Brasil. andre.cunha@ufrgs.br 


\section{I}

\section{Introducción}

El Brasil es uno de los países con mayor desigualdad del mundo. Sin embargo, desde el comienzo de este nuevo siglo, la desigualdad de ingresos en el Brasil sigue una trayectoria de reducción lenta pero casi continua, ya se mida por medio del coeficiente de desigualdad de Gini o mediante la relación de los ingresos correspondientes a los distintos estratos de la distribución (Barros, De Carvalho y Mendonça, 2010). Otro aspecto significativo vinculado a esta disminución de la desigualdad consiste en su coincidencia con un ciclo de expansión de los ingresos con relativa estabilidad macroeconómica, caracterizada —entre otras cosas- por una inflación moderada, la tendencia a la disminución de la deuda neta del sector público y el incremento de la solvencia externa. Además, han crecido la oferta neta de empleos, su formalización y los salarios reales, al tiempo que ha retrocedido la pobreza económica (IPEA, 2010; FerrariFilho, Cunha y Bichara, 2014).

Las causas de esta disminución de la desigualdad de los ingresos son múltiples. Entre ellas, destacan la persistencia de la estabilidad económica (Rocha, 2000), los programas de transferencias condicionadas del gobierno federal y la evolución favorable de los ingresos del trabajo (Barros, De Carvalho y Mendonça, 2007 y 2010). Ese comportamiento virtuoso del mercado laboral podría estar vinculado a la pronunciada creación de empleos formales, la optimización del capital humano, la reducción de la discriminación salarial por razones de género y color de la piel y la disminución de la segmentación sectorial y geográfica.

La relevancia del mercado de trabajo en el proceso de desarrollo no es una novedad en el análisis económico, pero no ha sido muy destacada, especialmente en los países en desarrollo. El mercado de trabajo es el factor clave que explica cómo se reparte el crecimiento en la sociedad. La distribución de los beneficios del crecimiento está estrechamente ligada a la cantidad y calidad de los empleos creados durante el período de expansión (Paci y Serneels, 2007). No obstante, también resulta

$\square$ Este trabajo se desarrolló en el ámbito del Programa de Fomento a Pesquisa em Desenvolvimento Econômico (PDE) - Banco de Desarrollo del Brasil (BNDES), publicación de 2010, en el proyecto denominado "El nuevo ciclo de crecimiento de la economía brasileña: movilidad ocupacional y desigualdad de los ingresos". significativa la evolución temporal de esa estructura, es decir, la movilidad en el mercado laboral. Saber cómo puede acceder el trabajador a empleos mejor remunerados es fundamental para comprender la forma en que se distribuyen los ingresos del crecimiento económico. En ese sentido, es necesario entender los factores que determinan la movilidad ocupacional, las barreras existentes, la prima salarial de la movilidad y los efectos de esa movilidad en la distribución de los ingresos del trabajo. Ese es el objetivo fundamental de este artículo.

Existen indicios de que los trabajadores pueden utilizar la movilidad ocupacional para obtener aumentos salariales y también como forma de salir de situaciones precarias de trabajo, como la informalidad o una elevada incidencia de desempleo involuntario (Holzer, Lane y Vilhuber, 2003; García Pérez y Rebollo Sanz, 2005; Davia, 2006). Por otra parte, esos beneficios pueden ser nulos o limitados cuando existen barreras impuestas por la segmentación del mercado laboral. En el Brasil, aparte de los trabajos de Oliveira y Machado (2000) y Pinto y Neri (2000), que encontraron diferencias en el retorno de la movilidad entre los diversos grupos de población, existen pocos datos que muestren adecuadamente la repercusión de la movilidad ocupacional en la desigualdad de los ingresos. Por lo tanto, todavía no está claro si la movilidad ocupacional ha contribuido o no a lograr que aminore la desigualdad, sobre todo durante el período más reciente. Para contribuir al debate, este artículo tiene como objetivo analizar los factores que influyen en la movilidad ocupacional y verificar si esta supone un progreso socioeconómico y cuáles son sus efectos en la desigualdad de los ingresos en el Brasil, utilizando los datos de la Encuesta Mensual de Empleo para el período comprendido entre 2002 y 2010.

En particular, se pretende analizar el efecto de la movilidad en el diferencial de los salarios, en términos de la segmentación del mercado de trabajo (que determina las diferencias relativas al progreso socioeconómico y ocupacional, por ejemplo) y de las diferencias en el retorno de la movilidad, observando si los movimientos ocupacionales alteran o no la distribución de los ingresos, reduciendo el diferencial de los salarios. Se aportan nuevos datos sobre el efecto del capital humano y un análisis del destino socioeconómico de los trabajadores, que permiten constatar si la movilidad se está utilizando para avanzar en la estructura social y en la distribución 
de los ingresos o si la distribución está limitada por la segmentación del mercado de trabajo. Este enfoque de la movilidad y de la desigualdad permite evaluar la magnitud del grupo de trabajadores que, a pesar de los cambios ocupacionales que llevan a cabo, no consiguen salir de una estructura de reducido incremento salarial y tecnológico, con menor protección institucional. Después de esta Introducción, en la sección II se presenta una breve revisión de la bibliografía sobre la relación entre la movilidad ocupacional y los salarios. A continuación, en la sección III se exponen la metodología, los datos, los resultados y el correspondiente análisis. Por último, en las consideraciones finales de la sección IV se resumen los principales resultados.

\section{II}

\section{Revisión de la bibliografía: datos empíricos y sus consecuencias analíticas}

Los datos empíricos acerca de la movilidad ocupacional y sus efectos en el salario son abundantes, especialmente con respecto a los Estados Unidos de América y, más recientemente, en relación con los países europeos, además de algunos de América Latina (Beccaria y Maurizio, 2003). En el trabajo de Paci y Serneels (2007), centrado en la relación entre el mercado de trabajo y el desarrollo, se considera la estructura del mercado laboral y la movilidad ocupacional como variables clave para explicar la distribución de los ingresos. En el caso del Brasil, destacan los estudios de Pinto y Neri (2000) y de Oliveira y Machado (2000), en que se utilizaron los datos longitudinales de la Encuesta Mensual de Empleo del Instituto Brasileño de Geografía y Estadística (IBGE).

En la mayoría de los estudios empíricos se subraya el papel de la distribución ocupacional de los trabajadores como una herramienta esencial para explicar los salarios. Por lo tanto, los factores sociales y laborales, la discriminación y las diferencias de inversión o de acceso al capital humano son temas inherentes al debate sobre la distribución de ingresos en el Brasil. Según ese enfoque, el mercado de trabajo simplemente refleja las desigualdades sociales existentes en el país (Barros y Mendonça, 1995), además de actuar como creador de desigualdades, ya que se caracteriza por la presencia de segmentación y de discriminación de género y raza (Oliveira y Ribeiro, 1998; Oliveira, 1998 y 2003; Soares y Oliveira, 2004; Matos y Machado, 2006). Sin embargo, el mercado laboral brasileño también puede funcionar como una salida para situaciones de nivel muy bajo de ingresos o de pobreza (Barros, Machado y Mendonça, 1997).

En la bibliografía internacional se recalca el papel del capital humano, tanto el específico como el general, en la explicación de la movilidad ocupacional y del resultado salarial vinculado a ella. Booth y Francesconi (1999), por ejemplo, observan que la antigüedad en el mercado laboral tiene un efecto negativo en la movilidad de los trabajadores británicos y que el tiempo de experiencia específica en el empleo está negativamente correlacionado con los cambios internos. En el Brasil, se encontraron los mismos resultados (Orellano y Picchetti, 2001; Menezes-Filho, 2004; Flore y Menezes-Filho, 2008). Sin embargo, aparentemente la educación superior puede reducir la probabilidad de movilidad (Kambourov y Manovskii, 2004). Este resultado también se encuentra al considerar el capital humano específico, representado por la formación recibida dentro de la empresa, como consecuencia del aumento del costo de oportunidad (Dolton y Kidd, 1998).

Además, la movilidad ocupacional depende en apariencia del sector de actividad económica que se considera y del nivel de intensidad tecnológica (Zimmermann, 1998), y puede relacionarse con el tamaño de la empresa (Cheng y Kalleberg, 1996). En las investigaciones realizadas, se han encontrado diferencias en los patrones de movilidad de los diversos grupos de población, géneros, razas y niveles de ingresos. En general, pareciera que la tasa de movilidad es mayor entre los hombres que entre las mujeres (Gabriel, 2003; Parrado y Wolff, 1999) y que los grupos con ingresos más altos son más estables (Parrado, Caner y Wolff, 2007). También se observa alguna limitación en la movilidad ocupacional para empleos con altos salarios, ya que para estos el movimiento estaría limitado por la discriminación, la información parcial, las deficientes redes de empleo y la preferencia de los actores (Holzer, Lane y Vilhuber, 2003).

Para los fines de este artículo, cabe destacar el efecto de la movilidad ocupacional en el salario. En 
este sentido, la movilidad parece contribuir a mejorar la distribución de los ingresos (Fitzenberger y Kunze, 2005) e incluso puede incrementar la tasa de crecimiento salarial (García Pérez y Rebollo Sanz, 2005). Como se ha destacado anteriormente, existen muy pocos estudios sobre la movilidad ocupacional y sus efectos en la distribución de los ingresos en los países en desarrollo. Un buen ejemplo es el trabajo de Paci y Serneels (2007), donde se presentan resultados para algunos países en desarrollo y se muestra que existen significativas barreras para la movilidad ocupacional en los países de Oriente Medio y del norte de África, según el análisis de la movilidad entre segmentos formales e informales. Estos autores también señalan que existen grandes barreras para la movilidad ascendente de los trabajadores autónomos en México, determinadas por la educación y el acceso al capital. En otro estudio sobre Etiopía, Ghana y la República Unida de Tanzanía, analizaron el efecto de la movilidad entre sectores, definidos según el estatus ocupacional, y encontraron que hay fuertes barreras para la movilidad y que el factor principal del incremento salarial, cuando existe movilidad, es el tamaño de la empresa.

Como en los casos anteriores, una característica significativa del mercado de trabajo brasileño es la segmentación entre trabajadores formales e informales. Esta segmentación supone claras diferencias, ya que la movilidad ocupacional es menor entre los trabajadores formales y, especialmente, entre los que tienen más experiencia (Neri y otros, 1997). Por otra parte, el patrón de movilidad es diferente dependiendo del género y de la raza, de forma que las mujeres y las personas de raza negra en el Brasil están sobrerrepresentados en empleos de baja remuneración y en trayectorias laborales generalmente desfavorables, como por ejemplo, la movilidad descendente y la reducción salarial (Oliveira y Machado, 2000; Pinto y Neri, 2000). Para estos grupos, por lo tanto, la movilidad sería un sinónimo de simple rotación de la mano de obra, con cambios frecuentes de empleo y sin agregación de calificación ni aumento de la productividad.

De este modo, se constata que la estructura ocupacional es relevante para explicar la brecha salarial. La manera en que los trabajadores cambian de empleo o consiguen salir de un determinado segmento ocupacional puede alterar la configuración de la distribución de los ingresos. Por otra parte, la segmentación y la segregación ocupacionales son fenómenos que limitan la movilidad de los trabajadores y provocan, entre otras cosas, que un cambio de empleo solo reproduzca a lo largo del tiempo la estructura desigual de asignación de la mano de obra y no suponga progresos en la condición social del individuo (Fitzenberger y Kuzne, 2005; Maltseva, 2005). Es decir, la desigualdad salarial puede estar influida tanto por la estructura ocupacional como por los movimientos de los trabajadores que alteran esa estructura. Sin embargo, como se mostró en la revisión de la bibliografía, todavía no está claro el papel que ha desempeñado la movilidad ocupacional durante el período reciente de la economía brasileña. En ese sentido, en el presente artículo se procura avanzar en la comprensión de la movilidad ocupacional y sus efectos en la estructura socioeconómica de los trabajadores y en la distribución de los ingresos en el Brasil. También se procura determinar si la movilidad se ha utilizado para que el trabajador progrese dentro de la distribución de los ingresos o si únicamente está reproduciendo un mercado de trabajo segmentado.

\section{III}

\section{Datos y metodología}

Para avanzar hacia el objetivo de este estudio se han utilizado los microdatos de la Encuesta Mensual de Empleo, que permiten comparar la situación de los trabajadores en lo relativo a sus características laborales, su nivel de educación, su edad o su salario, por medio de un panel de datos mensuales en seis regiones metropolitanas del país: Belo Horizonte, Porto Alegre, Recife, Río de Janeiro, Salvador y São Paulo. La estrategia econométrica se divide en dos etapas. En la primera, se analizan los factores de la movilidad entre empleos o segmentos ocupacionales y, en la segunda, se estiman los efectos de esta movilidad en la distribución de los ingresos.

El esquema en forma de panel de la Encuesta Mensual de Empleo permite comparar la información sobre el empleo que tenía el individuo en dos puntos distintos del tiempo, de modo que pueden obtenerse cuatro tipos de resultados con respecto a los trabajadores con empleo, sin tener en cuenta a las personas que se salen de la muestra: 
i) el individuo tenía el mismo empleo en los dos períodos;

ii) tenía dos empleos diferentes;

iii) estaba desempleado, $\mathrm{y}$

iv) se hallaba inactivo.

La tasa de movilidad ocupacional expresa el porcentaje de trabajadores con empleo que, en cada período, tenían un empleo distinto del declarado anteriormente. De esta forma, la primera parte de la investigación consiste en determinar la probabilidad de que un individuo cambie de empleo.

Los empleos se clasifican en grupos de estatus sociolaboral, con una metodología inspirada en el trabajo de Jannuzzi (2004), tomando como base los promedios del salario por hora, el nivel de educación, la informalidad y la similitud entre actividades, como se muestra en el cuadro 1. Esa clasificación puede utilizarse para describir la estructura sociolaboral del mercado de trabajo brasileño y también para analizar la movilidad ocupacional (ascendente o descendente) en términos socioeconómicos (salario y estatus social) de los trabajadores.

Para captar esos múltiples cambios, en el estudio se estima un modelo logit multinomial, que amplía los modelos de opción binaria, dado por:

$$
\operatorname{Pr}\left(Y_{i}=j\right)=\frac{e^{\beta_{j}^{\prime} x_{i}}}{1+\sum_{k=0}^{2} e^{\beta_{j}^{\prime} x_{i}}}, \quad j=0,1,2
$$

donde $\operatorname{Pr}$ representa la probabilidad de que el individuo pase por el evento $j ; x_{i}$ es el vector de variables explicativas, y $\beta$ es el vector de parámetros que se desea estimar. El modelo ha sido utilizado, por ejemplo, por Budría y
Pereira (2004), para analizar la probabilidad de que los trabajadores de Portugal participen en programas de formación, y por Souza y Lima (2011), para investigar la probabilidad de que una persona esté desempleada, tenga un empleo formal o tenga un empleo informal en el Brasil.

Como variables explicativas de la probabilidad de realizar un ascenso o un descenso sociolaboral se utilizan factores demográficos, de capital humano y del mercado de trabajo, y se estima el siguiente modelo para captar elementos de los dos principales grupos teóricos de explicación de la movilidad (capital humano y mercados segmentados):

$$
\begin{gathered}
\operatorname{Pr}\left(Y_{i}=j\right)=f\left(\text { sexo, color, jefe, edad, } \sum\right. \text { calificación, } \\
\text { formal, } \left.\sum \text { sector, } \sum \text { región, } \sum \text { año }\right)
\end{gathered}
$$

donde $y$ toma el valor 0 si el trabajador permanece en la misma categoría sociolaboral en los dos períodos considerados, 1 si asciende de categoría y 2 si pasa a una categoría inferior; sexo es una variable binaria que designa el género; color toma el valor 1 para las personas de raza blanca y 0 para los demás casos; jefe es una variable ficticia que capta la condición del trabajador en la familia; edad es la edad del trabajador; calificación representa el nivel de educación ${ }^{1}$; formal es una variable binaria de valor 1 en el caso de los trabajadores formales;

\footnotetext{
${ }^{1}$ Sin calificación (hasta 3 años de estudio); baja calificación (4 a 7 años de estudio); semicalificados (8 a 10 años de estudio), y calificados (más de 10 años de estudio).
}

\begin{tabular}{|c|c|c|}
\hline Estatus sociolaboral & $\begin{array}{c}\text { Promedio de salario } \\
\text { por hora } \\
\text { (en reales })\end{array}$ & $\begin{array}{l}\text { Promedio de nivel } \\
\text { educativo } \\
\text { (en años de estudio) }\end{array}$ \\
\hline
\end{tabular}

1. Superior $\quad 14,50 \quad 10,18$

Directivos; directores de empresa; técnicos de laboratorio; profesionales de nivel superior; profesionales de la navegación, y supervisores.

Técnicos de nivel medio en general; joyeros y orfebres; supervisores industriales; operarios industriales; técnicos de navegación; trabajadores de transporte y vendedores.

Trabajadores de hotelería, alimentación, seguridad, entre otros; supervisores y trabajadores agrícolas; trabajadores comerciales y residenciales; empleados domésticos; vendedores ambulantes; reponedores comerciales y servicios personales.

Fuente: Encuesta Mensual de Empleo, 2002-2010. 
sector representa un conjunto de variables ficticias para los sectores de actividad económica ${ }^{2}$; región corresponde a variables ficticias para las seis regiones metropolitanas, y año se refiere a las variables ficticias de efectos anuales.

Después de haber estimado los factores de la movilidad ocupacional, se analizan —a través de regresiones cuantílicas (Koenker y Basset, 1978)—el efecto de la movilidad en el salario y la distribución de los ingresos salariales. Las regresiones cuantílicas permiten comparar los efectos marginales, o primas salariales de los factores, de los trabajadores más ricos y de los más pobres dentro de la distribución del salario. De esta manera, es posible verificar si la movilidad tiene un efecto distinto según el nivel de ingresos del individuo. Si los trabajadores más pobres tienen, por ejemplo, primas más elevadas por ascender sociolaboralmente, entonces la movilidad puede contribuir a reducir la diferencia salarial entre los diversos estratos de ingresos. Para determinar si estas diferencias son reales y significativas, es posible utilizar regresiones intercuantílicas (Koenker, 2000).

El modelo estimado, tanto para las regresiones condicionales a los cuantiles como para las intercuantílicas, viene dado por la siguiente ecuación:

$$
\begin{aligned}
& \ln \left(w_{2}\right)=f\left(\text { sexo, color, jefe, edad, edad }{ }^{2}, \sum\right. \text { calificación, } \\
& \text { formal, } \left.\sum \text { sector, } \sum \text { región, } \sum \text { año, ascenso, descenso }\right)
\end{aligned}
$$

donde $w_{2}$ es el salario recibido en el segundo período (después de la decisión de cambiar o no de empleo);

\footnotetext{
2 Sectores de productos básicos; industria; construcción; terciario y otras actividades.
}

sexo, color, jefe, edad, calificación, formal, sector, región y año se definen de la misma forma que se señaló anteriormente; "ascenso" y "descenso" son variables ficticias que marcan la dirección de la movilidad de los trabajadores, tomando como referencia a aquellos que cambiaron de segmento sociolaboral. De esta forma, dentro del modelo de regresión intercuantílica, si la movilidad tiene un efecto negativo significativo, eso supone que los movimientos ocupacionales contribuyen a reducir la diferencia salarial entre los cuantiles considerados $\mathrm{y}$, por lo tanto, reducen la desigualdad. Los cuantiles analizados son el $10^{\circ}$, el $25^{\circ}$, el $75^{\circ}$ y el $90^{\circ}$, ya que los dos primeros tienen la función de captar los ingresos de los trabajadores más pobres, mientras que los dos últimos representan a los individuos con salarios por hora más elevados.

El período de análisis corresponde al lapso comprendido entre 2002 y 2010 , utilizando una muestra de trabajadores asalariados del sector privado (incluidos los empleados del hogar) que tienen empleo en la semana de referencia, con una edad entre 18 y 65 años. La movilidad se analiza al comparar la información de la cuarta y octava entrevistas y los paneles se combinan según la propuesta de Ribas y Soares (2008). Los valores monetarios se convirtieron a reales de diciembre de 2010, de acuerdo con lo indicado por Corseuil y Foguel (2002). En total, la base contiene 79.736 observaciones, como se indica en el cuadro 2 , donde también se presentan algunas breves estadísticas sobre la base de datos utilizada. La matriz de varianza de cada modelo se estimó mediante la técnica de bootstrap, para garantizar el control de la heterocedasticidad (Buchinsky, 1998). A continuación, se muestran los resultados del análisis econométrico y se estudian sus consecuencias analíticas.

CUADRO 2

Brasil: promedio de estadísticas descriptivas, 2002-2010

\begin{tabular}{|c|c|c|c|}
\hline & Promedio & $25 \%$ más pobres & $25 \%$ más ricos \\
\hline Trabajadores que cambiaron de empleo & 29,0 & 27,7 & 32,4 \\
\hline Trabajadores que cambiaron de segmento & 15,3 & 12,6 & 19,7 \\
\hline Salario por hora en el segundo período (en reales) & 6,2 & 2,3 & 14,4 \\
\hline Hombres & 59,9 & 45,0 & 70,9 \\
\hline Personas de raza blanca & 54,7 & 38,6 & 73,3 \\
\hline Jefes de familia & 51,9 & 40,4 & 63,9 \\
\hline Edad media & 35,7 & 34,8 & 37,9 \\
\hline \multicolumn{4}{|l|}{ Nivel de calificación } \\
\hline Sin calificación (hasta 3 años de estudio) & 7,0 & 12,6 & 1,6 \\
\hline Baja calificación (4 a 7 años de estudio) & 23,2 & 33,0 & 8,6 \\
\hline Semicalificados ( 8 a 10 años de estudio) & 19,7 & 23,7 & 10,6 \\
\hline Calificados (más de 10 años de estudio) & 50,1 & 30,7 & 79,3 \\
\hline Tasa de formalidad & 79,3 & 66,6 & 87,6 \\
\hline
\end{tabular}

(En porcentajes, reales y años de estudio) 


\begin{tabular}{|c|c|c|c|}
\hline & Promedio & $25 \%$ más pobres & $25 \%$ más ricos \\
\hline \multicolumn{4}{|l|}{ Sector de actividad económica } \\
\hline Sector de productos básicos & 9,0 & 8,8 & 8,2 \\
\hline Industria & 14,3 & 6,1 & 23,4 \\
\hline Construcción & 5,4 & 5,7 & 4,4 \\
\hline Terciario & 70,1 & 78,2 & 62,1 \\
\hline Otras actividades & 1,3 & 1,2 & 2,0 \\
\hline \multicolumn{4}{|l|}{ Regiones metropolitanas } \\
\hline Recife & 5,8 & 10,8 & 2,7 \\
\hline Salvador & 7,7 & 12,8 & 6,0 \\
\hline Belo Horizonte & 17,7 & 20,4 & 15,1 \\
\hline Río de Janeiro & 27,3 & 32,0 & 23,2 \\
\hline São Paulo & 25,65 & 13,3 & 36,7 \\
\hline Porto Alegre & 15,9 & 10,82 & 16,29 \\
\hline Número de observaciones & 79736 & 19992 & 19906 \\
\hline
\end{tabular}

Fuente: elaboración propia sobre la base de datos de la Encuesta Mensual de Empleo.

\section{IV}

\section{Resultados y análisis}

En esta sección se presentan los resultados del análisis del papel de la movilidad sobre el diferencial de los salarios con los datos de la Encuesta Mensual de Empleo y se muestran los resultados del modelo de probabilidad de cambio ocupacional y las regresiones de salario estimadas con regresiones cuantílicas.

\section{Los factores de la movilidad sociolaboral}

Las categorías sociolaborales utilizadas se basan en los promedios del salario por hora, el nivel de educación y la tasa de informalidad dentro de cada empleo, agrupados en tres niveles: superior, medio e inferior. Teniendo en cuenta que el mercado de trabajo es la principal fuente de ingresos y que el modo en que los trabajadores se integran en este mercado puede determinar su horizonte de oportunidades, se puede sugerir que estas categorías representan una visión general de la estructura socioeconómica del país. En el cuadro 3 se denota que la estructura sociolaboral permaneció relativamente estable en la década de 2000 y solo hay que subrayar el aumento de dos puntos porcentuales de los trabajadores en actividades del segmento inferior.

Considerando la clasificación original de los empleos en la Encuesta Mensual de Empleo, en promedio, casi el 30\% de los trabajadores tenía un empleo diferente al declarado inicialmente. Entre los que cambiaron de empleo se constató que: i) el 53\% pasó a otro segmento sociolaboral, y ii) el $47 \%$ permaneció en el mismo segmento. La tasa de movilidad entre los segmentos es, en promedio, del $15 \%$ para toda la muestra, ya que un $7,6 \%$ de los trabajadores mostró una movilidad ascendente, es decir, salió de un segmento ocupacional más bajo y pasó a uno mejor, mientras que un 7,7\% registró una movilidad descendente.

Los resultados del modelo de probabilidad plasmado en la ecuación (2) se exponen en el cuadro 4, tomando como referencia la situación de inmovilidad sociolaboral. Como las categorías se forman teniendo en cuenta, entre otros factores, el nivel educativo de los trabajadores, se prepararon dos modelos, con y sin las variables binarias de

CUADRO 3

Brasil: categorías sociolaborales, 2002-2009 (En porcentajes)

\begin{tabular}{lccc}
\hline Año & Superior & Mediana & Inferior \\
\hline 2002 & 11,5 & 42,0 & 46,4 \\
2003 & 11,0 & 40,9 & 48,1 \\
2004 & 11,0 & 39,8 & 49,2 \\
2005 & 11,0 & 40,5 & 48,5 \\
2006 & 10,8 & 41,3 & 47,8 \\
2007 & 10,4 & 40,9 & 48,7 \\
2008 & 11,6 & 40,4 & 48,0 \\
2009 & 10,3 & 40,3 & 49,4 \\
Total & 10,9 & 40,7 & 48,4 \\
\hline
\end{tabular}

Fuente: elaboración propia sobre la base de datos de la Encuesta Mensual de Empleo. 
Brasil: efectos marginales de la probabilidad de movilidad sociolaboral, 2002-2010

\begin{tabular}{|c|c|c|c|c|c|c|}
\hline & \multicolumn{3}{|c|}{ Modelo 1} & \multicolumn{3}{|c|}{ Modelo 2} \\
\hline & Inmovilidad & Ascenso & Descenso & Inmovilidad & Ascenso & Descenso \\
\hline Sexo & $\begin{array}{l}-0,0543^{\mathrm{a}} \\
(0,003)\end{array}$ & $\begin{array}{l}0,0262^{\mathrm{a}} \\
(0,002)\end{array}$ & $\begin{array}{l}0,0280^{\mathrm{a}} \\
(0,002)\end{array}$ & $\begin{array}{l}-0,0555^{\mathrm{a}} \\
(0,003)\end{array}$ & $\begin{array}{l}0,0272^{\mathrm{a}} \\
(0,002)\end{array}$ & $\begin{array}{c}0,0282^{a} \\
(0,002)\end{array}$ \\
\hline Color de la piel & $\begin{array}{l}-0,0126^{a} \\
(0,003)\end{array}$ & $\begin{array}{l}0,0065^{\mathrm{a}} \\
(0,002)\end{array}$ & $\begin{array}{l}0,0061^{\mathrm{a}} \\
(0,002)\end{array}$ & $\begin{array}{l}-0,0226^{\mathrm{a}} \\
(0,003)\end{array}$ & $\begin{array}{c}0,0112^{\mathrm{a}} \\
(0,002)\end{array}$ & \\
\hline Jefe de familia & $0,0136^{\mathrm{a}}$ & $-0,0107^{\mathrm{a}}$ & $-0,0029$ & $0,0177^{\mathrm{a}}$ & $-0,0122^{\mathrm{a}}$ & $-0,0055^{\mathrm{b}}$ \\
\hline Edad & $(0,000)$ & $\begin{array}{l}-0,0010^{\mathrm{a}} \\
(0,000)\end{array}$ & $\begin{array}{l}-0,0004^{\mathrm{a}} \\
(0,000)\end{array}$ & $\begin{array}{l}0,0021^{\mathrm{a}} \\
(0,000)\end{array}$ & $\begin{array}{l}-0,0013^{\mathrm{a}} \\
(0,000)\end{array}$ & $\begin{array}{l}(0,002) \\
-0,0007^{\mathrm{a}} \\
(0,000)\end{array}$ \\
\hline $\begin{array}{l}\text { Baja calificación } \\
\text { ( } 4 \text { a } 7 \text { años de estudio) } \\
\text { Semicalificados } \\
\text { ( } 8 \text { a } 10 \text { años de estudio) } \\
\text { Calificados } \\
\text { (más de } 10 \text { años de estudio) }\end{array}$ & $\begin{array}{l}-0,0185^{\mathrm{a}} \\
(0,007) \\
-0,0556^{\mathrm{a}} \\
(0,008) \\
-0,0792^{\mathrm{a}} \\
(0,006)\end{array}$ & $\begin{array}{c}0,0077 \\
(0,005) \\
0,0220^{\mathrm{a}} \\
(0,006) \\
0,0337^{\mathrm{a}} \\
(0,005)\end{array}$ & $\begin{array}{l}0,0108^{\mathrm{b}} \\
(0,005) \\
0,0336^{\mathrm{a}} \\
(0,006) \\
0,0455^{\mathrm{a}} \\
(0,005)\end{array}$ & & & \\
\hline Formal & $\begin{array}{c}0,0035 \\
(0,003)\end{array}$ & $\begin{array}{l}0,0093^{\mathrm{a}} \\
(0,002)\end{array}$ & $\begin{array}{l}-0,0128^{a} \\
(0,003)\end{array}$ & & & \\
\hline Industria & $\begin{array}{l}-0,0025 \\
(0,005)\end{array}$ & $\begin{array}{l}-0,0082^{a} \\
(0,003)\end{array}$ & $\begin{array}{l}0,0107^{\mathrm{a}} \\
(0,004)\end{array}$ & $\begin{array}{l}-0,0100^{\mathrm{b}} \\
(0,005)\end{array}$ & $\begin{array}{l}-0,0050 \\
(0,003)\end{array}$ & $\begin{array}{l}0,0150^{\mathrm{a}} \\
(0,004)\end{array}$ \\
\hline Construcción & $\begin{array}{l}0,0470^{\mathrm{a}} \\
(0,005)\end{array}$ & $\begin{array}{l}-0,0107^{\mathrm{a}} \\
(0,004)\end{array}$ & $\begin{array}{l}-0,0363^{\mathrm{a}} \\
(0,003)\end{array}$ & $\begin{array}{l}0,0543^{\mathrm{a}} \\
(0,005)\end{array}$ & $\begin{array}{l}-0,0152^{\mathrm{a}} \\
(0,004)\end{array}$ & $\begin{array}{l}-0,0392^{a} \\
(0,003)\end{array}$ \\
\hline Terciario & $\begin{array}{l}0,0610^{\mathrm{a}} \\
(0,005)\end{array}$ & $\begin{array}{l}-0,0309^{a} \\
(0,003)\end{array}$ & $\begin{array}{l}-0,0301^{\mathrm{a}} \\
(0,003)\end{array}$ & $\begin{array}{l}0,0580^{\mathrm{a}} \\
(0,005)\end{array}$ & $\begin{array}{l}-0,0300^{\mathrm{a}} \\
(0,003)\end{array}$ & $\begin{array}{l}-0,0280^{\mathrm{a}} \\
(0,003)\end{array}$ \\
\hline Otras actividades & $\begin{array}{l}-0,0282^{b} \\
(0,011)\end{array}$ & $\begin{array}{l}0,0258^{a} \\
(0,009)\end{array}$ & $\begin{array}{c}0,0024 \\
(0,007)\end{array}$ & $\begin{array}{l}-0,0300^{\mathrm{a}} \\
(0,011)\end{array}$ & $\begin{array}{c}0,0269^{a} \\
(0,009)\end{array}$ & $\begin{array}{c}0,0031 \\
(0,008)\end{array}$ \\
\hline Recife & $\begin{array}{l}-0,0493^{a} \\
(0,006)\end{array}$ & $\begin{array}{l}0,0250^{\mathrm{a}} \\
(0,005)\end{array}$ & $\begin{array}{l}0,0243^{\mathrm{a}} \\
(0,005)\end{array}$ & $\begin{array}{l}-0,0521^{a} \\
(0,006)\end{array}$ & $\begin{array}{c}0,0263^{\mathrm{a}} \\
(0,005)\end{array}$ & $\begin{array}{l}0,0259^{a} \\
(0,005)\end{array}$ \\
\hline Salvador & $\begin{array}{l}0,0113^{b} \\
(0,005)\end{array}$ & $\begin{array}{l}-0,0055 \\
(0,004)\end{array}$ & $\begin{array}{l}-0,0058 \\
(0,004)\end{array}$ & $\begin{array}{r}0,0057 \\
(0,005)\end{array}$ & $\begin{array}{l}-0,0030 \\
(0,004)\end{array}$ & $\begin{array}{l}-0,0027 \\
(0,004)\end{array}$ \\
\hline Belo Horizonte & $\begin{array}{l}-0,0127^{\mathrm{a}} \\
(0,004)\end{array}$ & $\begin{array}{l}0,0067^{\mathrm{b}} \\
(0,003)\end{array}$ & $\begin{array}{l}0,0060^{\mathrm{b}} \\
(0,003)\end{array}$ & $\begin{array}{l}-0,0105^{\mathrm{a}} \\
(0,004)\end{array}$ & $\begin{array}{c}0,0062^{\mathrm{b}} \\
(0,003)\end{array}$ & $\begin{array}{c}0,0042 \\
(0,003)\end{array}$ \\
\hline Río de Janeiro & $\begin{array}{c}0,0410^{\mathrm{a}} \\
(0,003)\end{array}$ & $\begin{array}{l}-0,0196^{\mathrm{a}} \\
(0,002)\end{array}$ & $\begin{array}{l}-0,0214^{\mathrm{a}} \\
(0,002)\end{array}$ & $\begin{array}{c}0,0434^{\mathrm{a}} \\
(0,003)\end{array}$ & $\begin{array}{l}-0,0208^{a} \\
(0,002)\end{array}$ & $\begin{array}{l}-0,0226^{a} \\
(0,002)\end{array}$ \\
\hline Porto Alegre & $\begin{array}{l}-0,0085^{b} \\
(0,004)\end{array}$ & $\begin{array}{l}0,0055^{\mathrm{c}} \\
(0,003)\end{array}$ & $\begin{array}{c}0,0030 \\
(0,003)\end{array}$ & $\begin{array}{l}-0,0014 \\
(0,004)\end{array}$ & $\begin{array}{r}0,0027 \\
(0,003)\end{array}$ & $\begin{array}{l}-0,0012 \\
(0,003)\end{array}$ \\
\hline Pseudo $\mathrm{R}^{2}$ & 0,0339 & & & 0,0286 & & \\
\hline $\begin{array}{l}\mathrm{N}^{\mathrm{o}} \text { de observaciones } \\
\chi^{2}\end{array}$ & $\begin{array}{c}79736 \\
2982,34\end{array}$ & & & $\begin{array}{c}79736 \\
2480,89\end{array}$ & & \\
\hline
\end{tabular}

Fuente: elaboración propia sobre la base de datos de la Encuesta Mensual de Empleo.

a $\mathrm{p}<0,01$.

b $\mathrm{p}<0,05$.

c $\mathrm{p}<0,10$.

Nota: variables ficticias anuales suprimidas. Desviaciones estándar robustas entre paréntesis.

calificación y de formalidad. Los resultados se expresan en términos de efectos marginales y, por razones de espacio, se omiten las variables ficticias anuales, si bien siguen estando disponibles a través de los autores (este procedimiento se utilizará también en los otros cuadros de resultados econométricos).

Los dos modelos estimados muestran resultados similares, de modo que se mantiene la coherencia entre los signos de los efectos marginales. Las mujeres tienen una probabilidad mayor de permanecer sin cambios en términos ocupacionales, mientras que los hombres son más flexibles tanto para la movilidad ascendente como para la descendente. Un efecto semejante se advierte al analizar el efecto de la variable ficticia relativa al color de la piel, ya que los trabajadores de raza blanca muestran mayor movilidad que los de otras razas, con resultados similares a los encontrados por Pinto y Neri (2000), que también utilizaron los datos de la Encuesta Mensual de Empleo, pero con respecto a la década de 1990. Entre los individuos que son jefes de familia, el signo positivo y el carácter significativo del efecto marginal en la probabilidad de inmovilidad puede indicar una 
mayor aversión al riesgo por parte de los responsables de asegurar el sustento familiar.

En relación con los factores de capital humano, la edad y la educación muestran signos contrarios, lo que confirma la hipótesis de que distintas formas de capital humano tienen efectos diferenciados en la movilidad ocupacional (Mincer y Jovanovic, 1979). La edad, que puede interpretarse como variable representativa de la experiencia y, por lo tanto, del capital humano específico, señala que los individuos más viejos tienden a permanecer en el mismo segmento ocupacional. En cambio, los trabajadores con mayor nivel educativo o capital humano general son más flexibles y cambian con más facilidad de segmento sociolaboral.

En promedio, los trabajadores formales e informales poseen una tasa similar de movilidad entre empleos, de alrededor del $29 \%$ para ambos grupos. Sin embargo, el modelo estimado deja ver que esa movilidad no conduce a resultados iguales cuando se consideran los destinos de los trabajadores. Los que tienen empleos formales y cambian de empleo suelen realizar movimientos ascendentes hacia ocupaciones en mejores segmentos, mientras que la movilidad de los trabajadores informales tiende a ser descendente con más frecuencia. En ese sentido, además de fomentar la estabilidad en el empleo, la posesión del contrato de trabajo podría estar promoviendo una protección adicional de las personas. La formalización del trabajador, como evidencian estos resultados, puede considerarse un factor que fomenta el progreso en la carrera profesional y, en consecuencia, en su estatus sociolaboral.

Estos resultados demuestran que la movilidad y la flexibilidad — que pueden entenderse, respectivamente, como el flujo de trabajadores entre empleos o segmentos y como el grado de libertad que ofrece el mercado para estos cambios y también para la contratación o el despido de la mano de obra- son dos temas estrechamente relacionados. Si, por una parte, el análisis corrobora los resultados, por ejemplo, de Barros y otros (1997), que ponen de manifiesto una considerable flexibilidad del mercado de trabajo en el país, por otra, ofrece información adicional al mostrar que el efecto de esa flexibilidad no es homogéneo entre grupos de trabajadores. Los asalariados informales que —en principio— son los más flexibles, no se están beneficiando de esa flexibilidad tanto como los trabajadores protegidos institucionalmente por el contrato de trabajo. Esta constatación permite sugerir que la eliminación o la disminución de la protección institucional no parecen coherentes, en este sentido, con políticas que procuran lograr una redistribución de los ingresos por medio del mercado laboral.
Tomando como verdadera la hipótesis de que la integración ocupacional es uno de los principales factores que determinan el nivel de ingresos, la manera y la velocidad con que los trabajadores pasan de un segmento ocupacional a otro también pueden indicar cambios en la distribución de los ingresos del país. En la subsección siguiente se presentan los resultados de la estimación de ecuaciones de salarios que captan el efecto de la movilidad sociolaboral a lo largo de los cuantiles de la distribución del salario por hora.

\section{Determinación del salario: regresiones cuantílicas}

Las regresiones cuantílicas forman la base de la determinación del retorno de la movilidad según estratos de ingresos. Se estimaron regresiones condicionales con respecto a los cuantiles $10^{\circ}, 25^{\circ}, 75^{\circ}$ y $90^{\circ}$. Los dos primeros tienen la función de captar a los trabajadores más pobres, mientras que los dos últimos representan a los individuos con salarios por hora más elevados.

Los resultados se pueden ver en el cuadro 5, que —además del modelo general— presenta también una estimación alternativa sin las variables de educación y formalidad, ya que estas forman la base de la agregación de los segmentos de ocupación y podrían estar demasiado correlacionadas con las variables ficticias de movilidad. Para cada característica personal o del mercado de trabajo, en los cuatro cuantiles condicionales se determinan los ingresos marginales por medio de la primera derivada aplicada a la ecuación de la distribución condicional del salario por hora.

Las variables típicas de las ecuaciones de Mincer presentan los signos esperados (Corseuil, 2002), pero con distintas intensidades en los diversos cuantiles condicionales. La variable binaria de género, por ejemplo, capta la diferencia entre hombres y mujeres en el mercado de trabajo, mientras que la variable relativa al color de la piel muestra el diferencial de salario entre personas de raza blanca y de otras razas. En ambos casos, los efectos marginales aumentan a medida que se avanza hacia la parte superior de la distribución condicional del salario por hora, lo que muestra que las diferencias entre los grupos demográficos se intensifican en los trabajadores más ricos. Con respecto a la calificación de los trabajadores, se pueden sacar dos conclusiones de los resultados estimados. Por una parte, tomando como referencia los trabajadores sin calificación, los efectos marginales son crecientes para mayores calificaciones. Por otra, los trabajadores de cuantiles más elevados registran mayores efectos marginales, es decir, más 
CUADRO 5

Brasil: ecuaciones cuantílicas de los salarios, 2002-2010

\begin{tabular}{|c|c|c|c|c|c|c|c|c|}
\hline \multirow{2}{*}{ Cuantiles } & \multicolumn{4}{|c|}{ Modelo 1} & \multicolumn{4}{|c|}{ Modelo 2} \\
\hline & $10^{\circ}$ & $25^{\circ}$ & $75^{\circ}$ & $90^{\circ}$ & $10^{\circ}$ & $25^{\circ}$ & $75^{\circ}$ & $90^{\circ}$ \\
\hline \multirow[t]{2}{*}{ Sexo } & $0,1216^{\mathrm{a}}$ & $0,1524^{\mathrm{a}}$ & $0,1991^{\mathrm{a}}$ & $0,1927^{\mathrm{a}}$ & $0,1333^{\mathrm{a}}$ & $0,1739^{a}$ & $0,2085^{\mathrm{a}}$ & $0,1844^{a}$ \\
\hline & $(0,0048)$ & $(0,0052)$ & $(0,0060)$ & $(0,0108)$ & $(0,0052)$ & $(0,0039)$ & $(0,0057)$ & $(0,0123)$ \\
\hline \multirow[t]{2}{*}{ Color de la piel } & $0,0821^{\mathrm{a}}$ & $0,1085^{a}$ & $0,2321^{\mathrm{a}}$ & $0,2749^{a}$ & $0,1056^{a}$ & $0,1468^{a}$ & $0,4381^{\mathrm{a}}$ & $0,7193^{a}$ \\
\hline & $(0,0048)$ & $(0,0045)$ & $(0,0068)$ & $(0,0084)$ & $(0,0046)$ & $(0,0037)$ & $(0,0073)$ & $(0,0091)$ \\
\hline \multirow[t]{2}{*}{ Jefe de familia } & $0,0754^{\mathrm{a}}$ & $0,0857^{a}$ & $0,1452^{a}$ & $0,1804^{a}$ & $0,0526^{a}$ & $0,0588^{a}$ & $0,1102^{\mathrm{a}}$ & $0,1714^{\mathrm{a}}$ \\
\hline & $(0,0042)$ & $(0,0050)$ & $(0,0060)$ & $(0,0092)$ & $(0,0053)$ & $(0,0045)$ & $(0,0081)$ & $(0,0146)$ \\
\hline \multirow[t]{2}{*}{ Edad } & $0,0054^{\mathrm{a}}$ & $0,0081^{\mathrm{a}}$ & $0,0172^{\mathrm{a}}$ & $0,0206^{\mathrm{a}}$ & $0,0013^{a}$ & $0,0029^{a}$ & $0,0095^{\mathrm{a}}$ & $0,0154^{\mathrm{a}}$ \\
\hline & $(0,0002)$ & $(0,0002)$ & $(0,0002)$ & $(0,0004)$ & $(0,0003)$ & $(0,0002)$ & $(0,0003)$ & $(0,0006)$ \\
\hline \multirow{2}{*}{$\begin{array}{l}\text { Baja calificación } \\
\text { (4 a } 7 \text { años de estudio) }\end{array}$} & $0,0982^{\mathrm{a}}$ & $0,1116^{\mathrm{a}}$ & $0,1459^{a}$ & $0,1508^{a}$ & & & & \\
\hline & $(0,0083)$ & $(0,0058)$ & $(0,0105)$ & $(0,0123)$ & & & & \\
\hline \multirow{2}{*}{$\begin{array}{l}\text { Semicalificados } \\
\text { ( } 8 \text { a } 10 \text { años de estudio) }\end{array}$} & $0,1773^{a}$ & $0,2017^{a}$ & $0,2968^{a}$ & $0,3310^{a}$ & & & & \\
\hline & $(0,0063)$ & $(0,0070)$ & $(0,0090)$ & $(0,0130)$ & & & & \\
\hline \multirow{2}{*}{$\begin{array}{l}\text { Calificados } \\
\text { (más de } 10 \text { años) }\end{array}$} & $0,3408^{a}$ & $0,4343^{a}$ & $0,8844^{\mathrm{a}}$ & $1,1918^{a}$ & & & & \\
\hline & $(0,0068)$ & $(0,0078)$ & $(0,0101)$ & $(0,0131)$ & & & & \\
\hline \multirow[t]{2}{*}{ Formal } & $0,2807^{\mathrm{a}}$ & $0,2116^{\mathrm{a}}$ & $0,1356^{\mathrm{a}}$ & $0,1116^{\mathrm{a}}$ & & & & \\
\hline & $(0,0060)$ & $(0,0058)$ & $(0,0054)$ & $(0,0098)$ & & & & \\
\hline \multirow[t]{2}{*}{ Industria } & $0,1270^{\mathrm{a}}$ & $0,1585^{\mathrm{a}}$ & $0,1993^{\mathrm{a}}$ & $0,1906^{\mathrm{a}}$ & $0,1514^{\mathrm{a}}$ & $0,1989^{a}$ & $0,3371^{\mathrm{a}}$ & $0,2929^{a}$ \\
\hline & $(0,0080)$ & $(0,0093)$ & $(0,0094)$ & $(0,0174)$ & $(0,0111)$ & $(0,0079)$ & $(0,0156)$ & $(0,0211)$ \\
\hline \multirow[t]{2}{*}{ Construcción } & $0,0633^{a}$ & $0,0617^{a}$ & $0,0677^{a}$ & $0,0855^{\mathrm{a}}$ & $-0,0674^{a}$ & $-0,0390^{a}$ & $-0,0295$ & $-0,0203$ \\
\hline & $(0,0106)$ & $(0,0096)$ & $(0,0142)$ & $(0,0207)$ & $(0,0130)$ & $(0,0095)$ & $(0,0198)$ & $(0,0325)$ \\
\hline \multirow[t]{2}{*}{ Terciario } & $-0,0174^{\mathrm{b}}$ & $-0,0042$ & $0,0118^{\mathrm{c}}$ & 0,0056 & $-0,0212^{b}$ & $0,0076^{\mathrm{b}}$ & $0,0491^{\mathrm{a}}$ & 0,0107 \\
\hline & $(0,0070)$ & $(0,0061)$ & $(0,0066)$ & $(0,0132)$ & $(0,0084)$ & $(0,0034)$ & $(0,0156)$ & $(0,0155)$ \\
\hline \multirow[t]{2}{*}{ Otras actividades } & $-0,0302$ & $0,0641^{\mathrm{a}}$ & $0,2412^{\mathrm{a}}$ & $0,2929^{a}$ & $-0,0975^{a}$ & $-0,0217$ & $0,4702^{a}$ & $0,5001^{a}$ \\
\hline & $(0,0257)$ & $(0,0241)$ & $(0,0392)$ & $(0,0490)$ & $(0,0223)$ & $(0,0345)$ & $(0,0505)$ & $(0,0698)$ \\
\hline \multirow[t]{2}{*}{ Recife } & $-0,3474^{\mathrm{a}}$ & $-0,3876^{a}$ & $-0,4731^{\mathrm{a}}$ & $-0,4999^{a}$ & $-0,3103^{a}$ & $-0,3484^{\mathrm{a}}$ & $-0,4691^{a}$ & $-0,5422^{a}$ \\
\hline & $(0,0124)$ & $(0,0087)$ & $(0,0135)$ & $(0,0180)$ & $(0,0145)$ & $(0,0066)$ & $(0,0156)$ & $(0,0282)$ \\
\hline \multirow[t]{2}{*}{ Salvador } & $-0,3163^{a}$ & $-0,3198^{a}$ & $-0,3210^{a}$ & $-0,3438^{a}$ & $-0,2797^{a}$ & $-0,2865^{a}$ & $-0,2120^{a}$ & $-0,1779^{a}$ \\
\hline & $(0,0070)$ & $(0,0074)$ & $(0,0113)$ & $(0,0168)$ & $(0,0117)$ & $(0,0063)$ & $(0,0168)$ & $(0,0273)$ \\
\hline \multirow[t]{2}{*}{ Belo Horizonte } & $-0,1583^{a}$ & $-0,1629^{a}$ & $-0,1832^{a}$ & $-0,2110^{a}$ & $-0,1302^{a}$ & $-0,1575^{a}$ & $-0,2166^{a}$ & $-0,2923^{a}$ \\
\hline & $(0,0066)$ & $(0,0048)$ & $(0,0084)$ & $(0,0134)$ & $(0,0054)$ & $(0,0044)$ & $(0,0111)$ & $(0,0187)$ \\
\hline \multirow[t]{2}{*}{ Río de Janeiro } & $-0,1647^{a}$ & $-0,1689^{a}$ & $-0,1956^{a}$ & $-0,2107^{a}$ & $-0,1686^{a}$ & $-0,1839^{a}$ & $-0,2396^{a}$ & $-0,3270^{a}$ \\
\hline & $(0,0063)$ & $(0,0031)$ & $(0,0090)$ & $(0,0109)$ & $(0,0044)$ & $(0,0051)$ & $(0,0151)$ & $(0,0142)$ \\
\hline \multirow[t]{2}{*}{ Porto Alegre } & $-0,0703^{a}$ & $-0,0998^{a}$ & $-0,2224^{a}$ & $-0,2685^{a}$ & $-0,0688^{a}$ & $-0,1052^{a}$ & $-0,3415^{a}$ & $-0,5461^{a}$ \\
\hline & $(0,0077)$ & $(0,0045)$ & $(0,0068)$ & $(0,0120)$ & $(0,0066)$ & $(0,0061)$ & $(0,0142)$ & $(0,0209)$ \\
\hline \multirow[t]{2}{*}{ Ascenso } & 0,0089 & $0,0197^{\mathrm{a}}$ & $0,0470^{\mathrm{a}}$ & 0,0121 & 0,0108 & $0,0362^{\mathrm{a}}$ & $0,1401^{\mathrm{a}}$ & $0,0996^{a}$ \\
\hline & $(0,0070)$ & $(0,0071)$ & $(0,0162)$ & $(0,0190)$ & $(0,0114)$ & $(0,0077)$ & $(0,0115)$ & $(0,0199)$ \\
\hline \multirow[t]{2}{*}{ Descenso } & $-0,0063$ & $-0,0031$ & $0,0277^{\mathrm{b}}$ & $-0,0212^{\mathrm{c}}$ & $-0,0003$ & $0,0183^{\mathrm{a}}$ & $0,1261^{\mathrm{a}}$ & $0,0877^{a}$ \\
\hline & $(0,0081)$ & $(0,0061)$ & $(0,0109)$ & $(0,0127)$ & $(0,0074)$ & $(0,0058)$ & $(0,0127)$ & $(0,0178)$ \\
\hline \multirow[t]{2}{*}{ Constante } & $0,0972^{\mathrm{a}}$ & $0,2104^{\mathrm{a}}$ & $0,3357^{\mathrm{a}}$ & $0,5075^{\mathrm{a}}$ & $0,6159^{a}$ & $0,7451^{\mathrm{a}}$ & $1,0699^{\mathrm{a}}$ & $1,3469^{a}$ \\
\hline & $(0,0137)$ & $(0,0141)$ & $(0,0147)$ & $(0,0215)$ & $(0,0145)$ & $(0,0100)$ & $(0,0243)$ & $(0,0334)$ \\
\hline Pseudo $\mathrm{R}^{2}$ & 0,1819 & 0,1749 & 0,2393 & 0,3025 & 0,1092 & 0,1041 & 0,1166 & 0,1515 \\
\hline $\mathrm{N}^{\mathrm{o}}$ de observaciones & 79736 & 79736 & 79736 & 79736 & 79736 & 79736 & 79736 & 79736 \\
\hline
\end{tabular}

Fuente: elaboración propia sobre la base de datos de la Encuesta Mensual de Empleo.

a $\mathrm{p}<0,01$.

b $\mathrm{p}<0,05$.

c $\mathrm{p}<0,10$.

Nota: variables ficticias anuales suprimidas. Desviaciones estándar robustas por bootstrap entre paréntesis. 
oportunidades o una mayor facilidad de transformar en salario la calificación adquirida. Se trata de resultados consistentes tanto con los primeros estudios que usaron regresiones cuantílicas en el Brasil, como el de Maciel, Campelo y Raposo (2001), como con los análisis más recientes, como el de Sampaio (2009). La posesión del contrato de trabajo parece perder fuerza para explicar el nivel de ingresos de los trabajadores con mayores salarios, ya que para ellos influyen otros factores más allá de la formalización del trabajo por medio del contrato (Pianto y Pianto, 2002).

Con respecto a la movilidad entre los segmentos sociolaborales, los signos positivos y el carácter significativo en la mayoría de los casos indican que, en el mercado de trabajo brasileño, la movilidad tiene el efecto de aumentar el salario de los individuos, incluso cuando se produce en dirección a un segmento sociolaboral inferior. Estos resultados revelan que cuando se comparan dos trabajadores, uno de los cuales cambió recientemente de empleo, mientras que el otro ha permanecido más tiempo en la misma actividad, el primero tiende a tener un salario por hora más elevado. En este sentido, la movilidad en el Brasil parece estar utilizándose como estrategia de aumento salarial, en lugar de la antigüedad o del tiempo de servicio en el empleo o en la categoría laboral (Fitzenberger y Kunze, 2005).

No obstante, el resultado positivo de la movilidad no debe considerarse necesariamente contradictorio con lo esperado según el tiempo de servicio. Albuquerque (2008) sugiere que, al menos en el caso de los trabajadores más jóvenes, la rotación se relaciona con los incrementos salariales, que también pueden interpretarse como un ascenso profesional. Por otra parte, el tiempo en un empleo igualmente tiene signo positivo, lo que indica que los trabajadores más experimentados suelen contar con mayores remuneraciones. En este sentido, podrían realizarse investigaciones más específicas por grupos de edad, para comparar el efecto de la movilidad entre los jóvenes que entran en el mercado de trabajo y otras personas de mayor edad y experiencia profesional.

Cuando se analizan los efectos a largo plazo de los cuantiles, la movilidad sociolaboral parece tener escasa repercusión en el salario de los trabajadores más pobres, pero adquiere mayor relevancia en los dos cuantiles intermedios de la distribución. Para los trabajadores más ricos, la movilidad —incluso descendente- supone una prima salarial positiva. Dentro del enfoque de los matches o ajustes ocupacionales (McLaughlin, 1991), los trabajadores con mayores salarios y, en general, con mejores empleos, solo deciden cambiar de empleo cuando esa decisión les proporciona beneficios, lo que demuestra la mayor presencia de cambios voluntarios en este grupo. Los resultados aquí presentados indican, pues, que la movilidad podría ser el efecto de la constatación por parte del trabajador de nuevos ajustes ocupacionales que dan lugar a una mayor productividad. Entre los más pobres, por otra parte, la frecuencia de la movilidad involuntaria puede ser mayor, como consecuencia de la percepción de la empresa de que la productividad del trabajador es menor de lo que se esperaba. En ese sentido, convendría realizar estudios adicionales para verificar si la movilidad de los más pobres proporciona menores beneficios, precisamente debido a una posible mayor incidencia de los cambios ocupacionales forzosos entre ellos.

Cuando los resultados se desagregan considerando el tipo de contrato de trabajo, en el cuadro 6 se denota que los efectos marginales de la movilidad sociolaboral solo son significativos para los trabajadores que tienen contrato de trabajo firmado, incluidos los situados en puntos más elevados de la distribución salarial. Entre los trabajadores informales, la movilidad sociolaboral

CUADRO 6

Brasil: efectos marginales de la movilidad para trabajadores formales e informales por cuantiles, 2002-2010

\begin{tabular}{|c|c|c|c|c|c|c|c|c|}
\hline \multirow{2}{*}{ Cuantiles } & \multicolumn{4}{|c|}{ Sectores formales } & \multicolumn{4}{|c|}{ Sectores informales } \\
\hline & $10^{\circ}$ & $25^{\circ}$ & $75^{\circ}$ & $90^{\circ}$ & $10^{\circ}$ & $25^{\circ}$ & $75^{\circ}$ & $90^{\circ}$ \\
\hline Ascenso & $\begin{array}{r}0,0183^{\mathrm{a}} \\
(0,0066)\end{array}$ & $\begin{array}{r}0,0325^{\mathrm{a}} \\
(0,0086)\end{array}$ & $\begin{array}{c}0,1404^{\mathrm{a}} \\
(0,0141)\end{array}$ & $\begin{array}{r}0,0912^{\mathrm{a}} \\
(0,0178)\end{array}$ & $\begin{array}{l}-0,0102 \\
(0,0333)\end{array}$ & $\begin{array}{c}0,0201 \\
(0,0178)\end{array}$ & $\begin{array}{r}0,0803^{b} \\
(0,0328)\end{array}$ & $\begin{array}{r}0,1337^{\mathrm{a}} \\
(0,0367)\end{array}$ \\
\hline Descenso & $\begin{array}{r}0,0165^{\mathrm{a}} \\
(0,0062)\end{array}$ & $\begin{array}{r}0,0408^{\mathrm{a}} \\
(0,0093)\end{array}$ & $\begin{array}{r}0,1379^{\mathrm{a}} \\
(0,0173)\end{array}$ & $\begin{array}{r}0,1059^{\mathrm{a}} \\
(0,0241)\end{array}$ & $\begin{array}{l}-0,0360 \\
(0,0224)\end{array}$ & $\begin{array}{l}-0,0146 \\
(0,0139)\end{array}$ & $\begin{array}{c}0,0313 \\
(0,0294)\end{array}$ & $\begin{array}{c}0,0464 \\
(0,0363)\end{array}$ \\
\hline
\end{tabular}

Fuente: elaboración propia sobre la base de datos de la Encuesta Mensual de Empleo.

a $\mathrm{p}<0,01$.

b $\mathrm{p}<0,05$.

Nota: desviaciones estándar robustas por bootstrap entre paréntesis. 
solo produce efectos positivos y significativos para los individuos con mayor salario por hora. Este resultado parece demostrar que los trabajadores informales, supuestamente los más flexibles, no se benefician financieramente de esa flexibilidad, de modo que se refuerza la idea de que, para este grupo, la movilidad es sinónimo de simple rotación de la mano de obra.

Estos análisis de los datos de la Encuesta Mensual de Empleo permiten no rechazar la hipótesis de que la movilidad entre empleos y segmentos ocupacionales tiene un efecto significativo en los salarios de los trabajadores, tal como se señala en Oliveira y Machado (2000). No obstante, la estimación al considerar tanto el análisis por cuantiles como el tipo de desagregación de los empleos añade nueva información al análisis empírico en el Brasil. Utilizando las regresiones cuantílicas, se descubren diferencias entre los cuantiles de la distribución del salario por hora. La movilidad tiene un efecto más relevante en los trabajadores con mayor remuneración y, dependiendo del nivel de desagregación, una repercusión prácticamente nula o negativa en los más pobres. Esto supone que la movilidad, a pesar de su capacidad de aumentar los ingresos, puede provocar un incremento de la distorsión de la distribución salarial y atenuar la caída esperada de la desigualdad de ingresos.

\section{Determinación del diferencial entre ricos y pobres: regresiones intercuantílicas}

Los resultados anteriores evidencian un valor más elevado del efecto marginal de la movilidad en los trabajadores con mayores ingresos. Teniendo en cuenta ese hecho, en esta subsección se presenta la estimación de regresiones intercuantílicas, que evalúan si esta diferencia entre los retornos marginales de dos cuantiles condicionales es o no significativa.

En el cuadro 7 se exponen los resultados para las diferencias entre los cuantiles $10^{\circ}$ y $90^{\circ}$ y entre los cuantiles $25^{\circ}$ y $75^{\circ}$. Los signos positivos, siempre que se trate de cantidades significativas, indican que el factor analizado tiene un mayor efecto marginal en el salario por hora de los cuantiles superiores. De esta forma, pueden interpretarse como factores que aumentan la diferencia salarial entre los dos cuantiles comparados, mientras que los signos negativos indican que el componente contribuye a que disminuya la desigualdad entre puntos de la distribución. En el caso de variables binarias, un signo positivo del coeficiente estimado puede interpretarse como un incremento del diferencial intercuantílico del salario por hora, en relación con la categoría de referencia.
Entre las variables utilizadas en los modelos, solo las variables ficticias regional y de formalidad presentan signos negativos, lo que indica que la diferencia entre los trabajadores más ricos y los más pobres podría estar reduciéndose a través de la convergencia de los salarios regionales y de la formalización, entre otras causas. En el caso de los empleos informales, este hecho puede ayudar a explicar la caída reciente de la desigualdad en el país, ya que el porcentaje de empleos informales también ha ido disminuyendo sistemáticamente (Mello y Santos, 2009). Esto ofrece una prueba más del papel desempeñado por la protección institucional, ante todo para equilibrar las diferencias entre los extremos de la distribución de ingresos. Una vez más, de este efecto se deduce la necesidad de realizar nuevos estudios sobre el papel de la segmentación entre empleos formales e informales del mercado de trabajo, quizás ampliando el análisis realizado por Curi y Menezes-Filho (2004), que investigaron la transición de trabajadores entre estos dos segmentos, el desempleo y la inactividad, pero sin analizar la repercusión que tienen esos desplazamientos en los ingresos laborales. Según los datos presentados en este estudio, puede plantearse la hipótesis de la existencia de una relación significativa entre la movilidad de empleos formales e informales y la desigualdad de ingresos.

Las variables que captan las transiciones entre los segmentos sociolaborales definidos en este estudio presentan signos positivos y significativos en la gran mayoría de los casos. Esto permite comprobar que el retorno o prima salarial de la movilidad es más alto entre los trabajadores de las franjas de ingresos superiores.

Si se consideran de manera conjunta con los datos analizados anteriormente, estos resultados dejan ver que la movilidad ha tenido un doble efecto en los salarios de los trabajadores, aumentando por una parte los ingresos y, por otra, contribuyendo a engrosar las diferencias salariales. En otras palabras, si se comparan dos grupos distintos de trabajadores, uno formado por personas que han cambiado recientemente de segmento ocupacional y otro con aquellos que se mantuvieron en la misma categoría durante ambos períodos de evaluación, el primer grupo presenta un salario por hora más alto, especialmente entre las personas cuyo cambio de empleo fue ascendente. Sin embargo, la prima salarial por este cambio, medida en términos del efecto marginal de las variables ficticias de movilidad, es significativamente más elevada entre los trabajadores con mayores ingresos en comparación con los que tienen un salario por hora correspondiente a la parte inferior de la distribución. Esto demuestra que los trabajadores de mayores ingresos se benefician más de los cambios de empleo que los más 
CUADRO 7

Brasil: regresiones intercuantílicas, 2002-2010

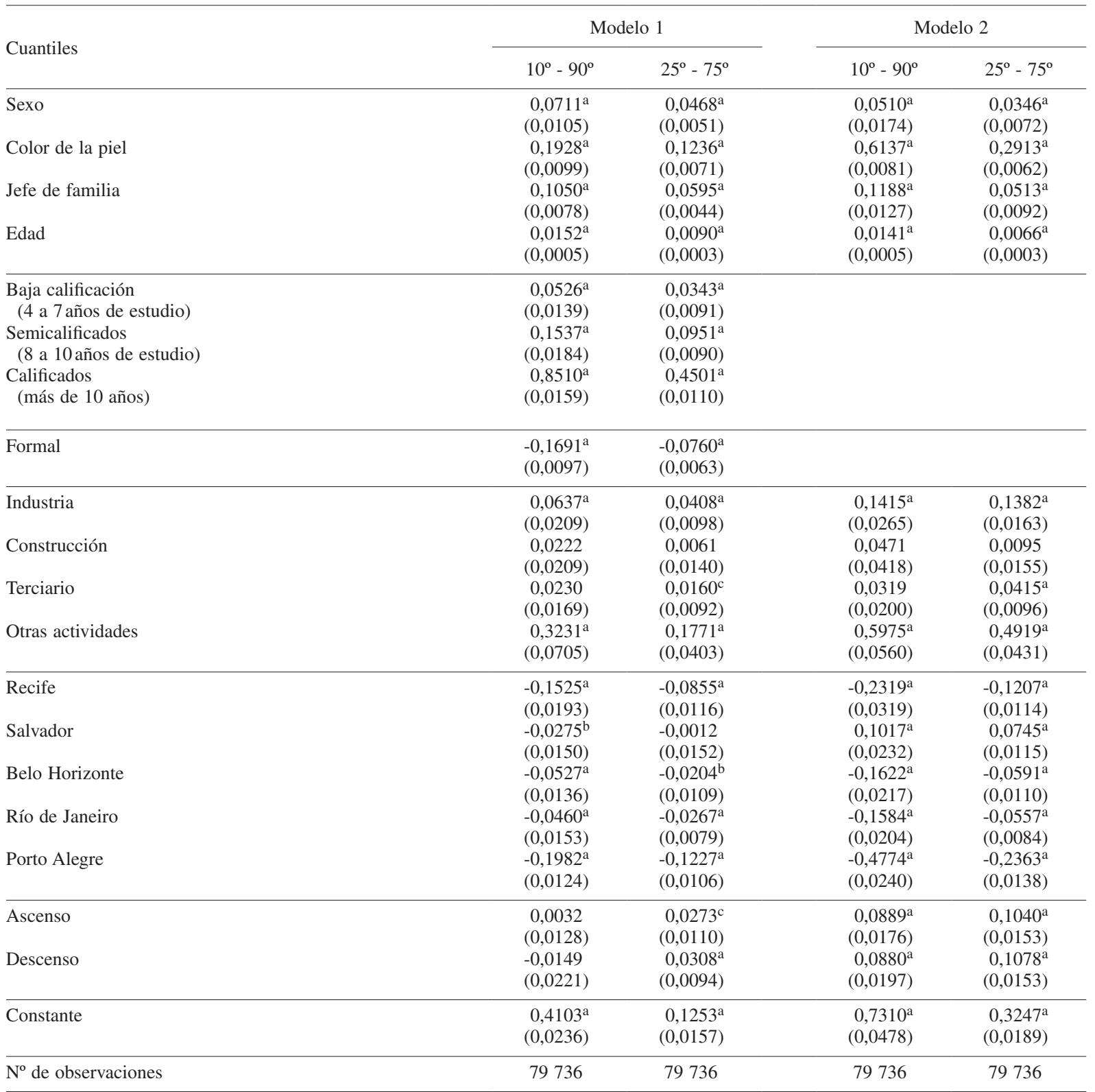

Fuente: elaboración propia sobre la base de datos de la Encuesta Mensual de Empleo.

a $\mathrm{p}<0,01$.

b $\mathrm{p}<0,10$

c $\mathrm{p}<0,05$.

Nota: variables ficticias anuales suprimidas. Desviaciones estándar robustas por bootstrap entre paréntesis. 
pobres, o que los primeros están en mejores condiciones para transformar ese cambio en una prima salarial, incluso cuando el cambio se produce hacia una categoría ocupacional inferior.

De este modo, si el retorno de la movilidad es distinto y más favorable a los trabajadores situados en la parte superior de la distribución de ingresos, la movilidad, tal como se ha producido hasta ahora, parece contribuir a un aumento de las diferencias salariales entre trabajadores ricos y pobres.

Si los resultados muestran que la movilidad entre segmentos fomenta el acrecentamiento de la diferencia entre los trabajadores que tienen mayores ingresos y los más pobres, ¿cómo se puede explicar la reciente disminución de la desigualdad? En realidad, estos hechos pueden considerarse como complementarios y no contradictorios, ya que la explicación más plausible consiste en que el mercado laboral en el país ha actuado como creador de desigualdades, limitando los efectos distributivos del ambiente macroeconómico más favorable de los dos últimos años. Una causa significativa de esta limitación, por lo tanto, consiste en la forma en que este mercado ha promovido los cambios de los trabajadores de un empleo a otro o de un segmento ocupacional a otro.

En el cuadro 8 se aprecian los coeficientes estimados cuando la muestra de trabajadores se divide en formales e informales. Se observa que los signos de la diferencia intercuantílica siguen siendo positivos, la mayoría de las veces con valores significativos, y solo varían en cuanto a la intensidad. La movilidad entre segmentos sociolaborales tiene un efecto mayor en la diferencia entre los extremos de la distribución del salario de los trabajadores informales. Estos resultados parecen indicar que la movilidad ocupacional entre los trabajadores con mayores ingresos podría utilizarse principalmente como estrategia para obtener mayores incrementos salariales, ya que solo se realiza cuando el impacto en el salario es suficientemente grande como para cubrir el costo de oportunidad de la estabilidad. En contrapartida, es posible que, entre los trabajadores más pobres, la movilidad se esté usando como estrategia de supervivencia social.

En síntesis, los resultados indican que la movilidad entre segmentos sociolaborales tiene un doble efecto en el mercado de trabajo. Por una parte, los trabajadores han utilizado este mecanismo para lograr aumentos salariales, como alternativa a la estabilidad y acumulación de capital humano específico. Esto confirma la hipótesis de que las transiciones ocupacionales pueden emplearse como forma de salir de situaciones de mayor riesgo o también como medio de avance profesional. Por otra, este uso no ha provocado una disminución de los diferenciales de salarios entre los distintos estratos de la distribución de los ingresos. Esto se debe al hecho de que los trabajadores con salarios más altos gozan de mejores condiciones para transformar la movilidad en incrementos salariales significativos.

Una posible explicación de este efecto podría ser que, entre los trabajadores con mayores salarios, la movilidad solo tendría lugar cuando sus beneficios superasen los costos del cambio, como la pérdida de antigüedad en el empleo anterior y otros costos de tipo no económico. Para estos trabajadores, el cambio de empleo o de segmento sociolaboral parece ser más bien el resultado de decisiones voluntarias, en la búsqueda de ajustes ocupacionales más productivos o con la intención de lograr un mayor retorno salarial. Entre los trabajadores más pobres, en cambio, especialmente entre los informales, la movilidad puede deberse a otros factores, quizás con un mayor componente involuntario, que obliguen al trabajador a cambiar a un empleo del mismo nivel sociolaboral que no represente un efecto marginal significativo (Hachen, 1992).

Brasil: coeficientes de movilidad de las regresiones intercuantílicas para trabajadores formales e informales

\begin{tabular}{|c|c|c|c|c|}
\hline & \multicolumn{2}{|c|}{ Cuantiles formales } & \multicolumn{2}{|c|}{ Cuantiles informales } \\
\hline & $10^{\circ}-90^{\circ}$ & $25^{\circ}-75^{\circ}$ & $10^{\circ}-90^{\circ}$ & $25^{\circ}-75^{\circ}$ \\
\hline Ascenso & $\begin{array}{c}0,0729^{\mathrm{a}} \\
(0,0199)\end{array}$ & $\begin{array}{r}0,1079^{a} \\
(0,0148)\end{array}$ & $\begin{array}{c}0,1439^{\mathrm{a}} \\
(0,0415)\end{array}$ & $\begin{array}{r}0,0602^{\mathrm{b}} \\
(0,0321)\end{array}$ \\
\hline Descenso & $\begin{array}{c}0,0894^{\mathrm{a}} \\
(0,0212)\end{array}$ & $\begin{array}{c}0,0971^{\mathrm{a}} \\
(0,0159)\end{array}$ & $\begin{array}{c}0,0824 \\
(0,0521)\end{array}$ & $\begin{array}{c}0,0459 \\
(0,0344)\end{array}$ \\
\hline
\end{tabular}

Fuente: elaboración propia sobre la base de datos de la Encuesta Mensual de Empleo.

a $\mathrm{p}<0,01$.

b $\mathrm{p}<0,10$.

Nota: desviaciones estándar robustas por bootstrap entre paréntesis. 
En consecuencia, esta movilidad, a pesar de contribuir a un aumento salarial, fomenta la existencia de una mayor diferencia entre los salarios de los trabajadores de los extremos de la distribución de los ingresos. Incluso si se tienen en cuenta los datos recientes que indican que la movilidad puede contribuir a reducir la diferencia salarial entre algunos grupos (hombres y mujeres, personas de raza negra y de raza blanca, entre otros) (Monsueto, 2008; Ruesga, Bichara y Monsueto, 2009), los resultados de este trabajo parecen indicar que esas reducciones ocurren dentro del mismo grupo de la distribución de los ingresos. Es decir, se produce una reducción del diferencial de salarios entre hombres y mujeres pobres y entre hombres y mujeres con mayor salario, pero no entre mujeres pobres y ricas, por ejemplo.

Los resultados parecen apuntar a la necesidad de un nuevo enfoque para las políticas públicas relativas al mercado de trabajo, de manera que se fortalezca el papel que juega la movilidad en el aumento salarial y se compense el efecto de incremento de las disparidades de ingresos. La creación de empleos debe privilegiar a los sectores en que la movilidad puede generar beneficios a los trabajadores, en particular a los de ingresos más bajos. En ese sentido, la reestructuración o la elaboración de nuevos programas podría basarse, por ejemplo, en los sistemas de "flexiseguridad" o flexibilidad con seguridad, adoptados de forma directa o indirecta por algunos países europeos (Auer, Berg y Coulibaly, 2005; Andersen y Svarer, 2006). Para eso, la economía debería funcionar de manera que concediese una cierta flexibilidad a las empresas a la hora de planear el volumen de mano de obra según los ciclos económicos, pero con un sistema de protección o de pensiones vinculado a los programas de actualización de la calificación profesional y de reubicación laboral. Al mismo tiempo, deben existir políticas dirigidas a mejorar las condiciones de trabajo en los empleos con estatus sociolaboral más bajo, como los incentivos para el aumento de la formalización y los incrementos reales de los ingresos por medio del salario mínimo. Como señalan Fontes, Pero y Berg (2012), estos segmentos suelen ser los más beneficiados por aumentos reales del salario mínimo, lo que puede contribuir a la reducción de la desigualdad de ingresos.

Además, cabe destacar que el análisis realizado en este artículo se refiere a un período con perspectivas favorables de empleo e incrementos reales de los salarios en la economía brasileña y que estos resultados pueden ser distintos en momentos de estancamiento económico, en los que la incidencia de movilidad involuntaria tiende a ser mayor, también entre los trabajadores formales, y se eleva la necesidad de tomar medidas de protección laboral. De la misma forma, como muestran Firpo y Reis (2007), la política de aumento real del salario mínimo definido por la legislación brasileña ha contribuido a aminorar la desigualdad. Esta política puede haber sido esencial para atenuar los efectos negativos que produce la movilidad ocupacional en las diferencias salariales entre los extremos de la distribución de los ingresos, limitando las pérdidas salariales de los trabajadores que realizaron cambios entre los empleos con estatus más bajo, es decir, los más afectados por la simple rotación de la mano de obra.

\section{V}

\section{Consideraciones finales}

Este trabajo parte de la hipótesis de que la desigualdad de salarios en el Brasil no es el resultado de un único factor, sino de un conjunto de factores relacionados, entre los que destaca el papel del mercado de trabajo y de la distribución ocupacional de los trabajadores. Si la forma en que está distribuida la mano de obra afecta a los salarios, entonces la movilidad ocupacional y entre segmentos debe ser capaz de alterar la distribución de los ingresos en el país. Así pues, la investigación tuvo como objetivo analizar el efecto de la movilidad en los salarios y en el diferencial de los ingresos entre los trabajadores más ricos y los más pobres, buscando datos sobre el papel que tuvo esa movilidad en la caída reciente de la desigualdad en el país. Se utilizaron los datos de la Encuesta Mensual de Empleo de los años 2002 a 2010 , mediante modelos de probabilidad de la movilidad entre segmentos de ocupación y regresiones cuantílicas sobre ecuaciones salariales de Mincer, empleando variables ficticias que captan los movimientos ascendentes y descendentes de los trabajadores.

El análisis ofrece puntos de discusión a través de nuevos resultados para el caso brasileño, los que muestran, por ejemplo, que los trabajadores más pobres presentan un menor efecto marginal positivo de la movilidad en 
comparación con la repercusión del mismo factor en los salarios de los individuos con mayor remuneración. Eso indica que la movilidad ocupacional, tal como se ha producido en el país, no genera los mismos beneficios para las distintas franjas de remuneración.

En consecuencia, se puede plantear la hipótesis de que, entre los trabajadores con mayor remuneración, la movilidad solo se produce cuando los beneficios del cambio superan sus costos, como la pérdida de capital humano específico y otros costos no económicos. Dicho de otra forma, los cambios voluntarios de empleo podrían ser más frecuentes entre esta parte de la mano de obra, como consecuencia de la búsqueda de ajustes ocupacionales más lucrativos. Entre los trabajadores más pobres, por el contrario, la hipótesis consiste en que los cambios entre segmentos y empleos son causados con más frecuencia por movimientos involuntarios o simple rotación de la mano de obra, de manera que los efectos en el salario no son significativos o incluso son negativos.

Por lo tanto, la movilidad de los trabajadores brasileños, por una parte, puede contribuir a los aumentos salariales, ya que presenta retornos marginales positivos. Sin embargo, también fomenta una mayor diferencia salarial entre los trabajadores en los extremos de la distribución de los ingresos. Si bien algunos datos recientes muestran que la movilidad puede contribuir a reducir las diferencias salariales entre algunos grupos (hombres y mujeres, personas de raza negra y de raza blanca, entre otros), como se observa en Ruesga, Bichara y Monsueto (2009), los resultados del presente trabajo parecen indicar que estas reducciones se producen dentro del mismo grupo de la distribución de los ingresos. Es decir, tiene lugar una disminución del diferencial de salarios entre hombres y mujeres pobres y entre hombres y mujeres con mayor salario, pero no entre mujeres pobres y ricas, por ejemplo. Además, los modelos de probabilidad de cambio ocupacional muestran que los trabajadores más flexibles, es decir, los informales, tienden a descender a segmentos con menor estatus sociolaboral cuando cambian de empleo. Eso supone que la eliminación o la reducción de la protección institucional no parecen ser coherentes con las políticas de redistribución de los ingresos por medio del mercado de trabajo.

Así, entender estos resultados parece fundamental para formular un conjunto de políticas de empleo eficientes que no solo mejoren la empleabilidad, sino que también promuevan el aumento de la calidad de vida de los trabajadores brasileños, reduciendo las desigualdades sociales. De esa forma, se podría fomentar un crecimiento óptimo de la productividad del trabajo y de la competitividad de toda la economía. Al mismo tiempo, una política de incremento del salario mínimo tiende a hacer que aminoren los efectos negativos de la movilidad ocupacional entre los trabajadores en empleos con menor estatus sociolaboral, es decir, los que tienen una remuneración más baja.

\section{Bibliografia}

Albuquerque, L.S.G. (2008), "Mobilidade de emprego entre os jovens brasileiros", Río de Janeiro, Universidad Federal de Río de Janeiro.

Andersen, T.M. y M. Svarer (2006), "Flexicurity-the Danish labour market model", Ekonomisk Debatt, $\mathrm{N}^{\circ} 1$.

Auer, P., J.E. Berg e I. Coulibaly (2005), “EEl trabajo estable mejora la productividad?", Revista Internacional del Trabajo, vol. 124, $\mathrm{N}^{\circ}$ 3, Ginebra, Organización Internacional del Trabajo (OIT).

Barros, R.P., A.F. Machado y R.S.P. Mendonça (1997), “A desigualdade da pobreza: estratégias ocupacionais e diferenciais por gênero", Texto para Discussão, N 453, Río de Janeiro, Instituto de Investigación Económica Aplicada (IPEA).

Barros, R.P. y R.S.P. Mendonça (1995), "Os determinantes da desigualdade no Brasil”, Texto para Discussão, $\mathrm{N}^{\circ} 377$, Río de Janeiro, Instituto de Investigación Económica Aplicada (IPEA).

Barros, R., M. de Carvalho y R.S.P. Mendonça (2010), "Determinantes da queda na desigualdade de renda no Brasil", Texto para Discussão, $\mathrm{N}^{\circ} 1460$, Río de Janeiro, Instituto de Investigación Económica Aplicada (IPEA).

(2007), "A queda recente da desigualdade de renda no Brasil", Texto para Discussão, № 1258, Río de Janeiro, Instituto de Investigación Económica Aplicada (IPEA).

Barros, R.P. y otros (1997), "Uma avaliação empírica do grau de flexibilidade alocativa do mercado de trabalho brasileiro", Texto para Discussão, $\mathrm{N}^{\circ} 499$, Río de Janeiro, Instituto de Investigación Económica Aplicada (IPEA).
Beccaria, L. y R. Maurizio (2003), "Movilidad ocupacional en Argentina", $6^{\circ}$ Congreso Nacional de Estudios del Trabajo, Buenos Aires, Asociación Argentina de Especialistas en Estudios del Trabajo.

Booth, A. y M. Francesconi (1999), "Job mobility in 1990s Britain: does gender matter?", ISER Working Paper Series, N 1999-26, Essex, Institute for Social and Economic Research.

Buchinsky, M. (1998), "Recent advances in quantile models: a practical guideline for empirical research", The Journal of Human Resources, vol. 33, $\mathrm{N}^{\circ} 1$, Madison, Wisconsin, University of Wisconsin Press.

Budría, S. y P.T. Pereira (2004), "On the returns to training in Portugal", CEEApLA, Working Paper Series, $\mathrm{N}^{\circ}$ 01/2004, Bonn, 2004.

Cheng, M.M. y A.L. Kalleberg (1996), "Labor market structures in Japan: an analysis of organizational and occupational mobility patterns", Social Forces, vol. 74, $\mathrm{N}^{\circ}$ 4, Oxford, Oxford University Press.

Corseuil, C.H. (ed.) (2002), Estrutura salarial: aspectos conceituais e novos resultados para o Brasil, Río de Janeiro, Instituto de Investigación Económica Aplicada (IPEA).

Corseuil, C.H. y M.N. Foguel (2002), "Uma sugestão de deflatores para rendas obtidas a partir de algumas pesquisas domiciliares do IBGE", Texto para Discussão, № 897, Río de Janeiro, Instituto de Investigación Económica Aplicada (IPEA). 
Curi, A.Z. y N.A. Menezes-Filho (2004), "Os determinantes das transições ocupacionais no mercado de trabalho brasileiro", Anais do XXXII Encontro Nacional de Economia, Río de Janeiro, Asociación Nacional de Centros de Posgrado en Economía (ANPEC).

Davia, M.A. (2006), "Studying the impact of job mobility on wage growth at the beginning of the employment career in Spain", documento presentado en el IX Encuentro de Economía Aplicada, Jaén.

Dolton, P.J. y M.P. Kidd (1998), "Job changes, occupational mobility and human capital acquisition: an empirical analysis", Bulletin of Economic Research, vol. 50, No 4, Blackwell Publishing.

Ferrari-Filho, F., A.M. Cunha y J.S. Bichara (2014), "Brazilian countercyclical economic policies as a response to the Great Recession: a critical analysis and an alternative proposal to ensure macroeconomic stability", Journal of Post Keynesian Economics, vol. 36, $\mathrm{N}^{\circ}$ 3, M.E. Sharpe.

Firpo, S. y M.C. Reis (2007), "O salário mínimo e a queda recente da desigualdade no Brasil", Desigualdade de renda no Brasil: uma análise da queda recente (volume 2), R.P. Barros, M.N. Foguel y G. Ulyssea (orgs.), Brasilia, Instituto de Investigación Económica Aplicada (IPEA).

Fitzenberger, B. y A. Kunze (2005), "Vocational training and gender: wages and occupational mobility among young workers", ZEW Discussion Papers, $N^{\circ}$ 05-66, Mannheim, Zentrum für Europäische Wirtschaftsforschung (ZEW).

Flore, P. y N. Menezes-Filho (2008), "Mobilidade entre ocupações e efeitos salariais", Anais do XXXVI Encontro Nacional de Economia, Río de Janeiro, Asociación Nacional de Centros de Posgrado en Economía (ANPEC).

Fontes, A., V. Pero y J. Berg (2012), "Low-paid employment in Brazil”, International Labour Review, vol. 151, N 3, Wiley.

Gabriel, P.E. (2003), "An examination of occupational mobility among full-time workers", Monthly Labor Review, vol. 129, $\mathrm{N}^{\circ}$ 9, Washington, D.C., Oficina de Estadísticas Laborales.

García Pérez, J.I. e Y. Rebollo Sanz (2005), "Wages changes through job mobility in Europe: a multinomial endogenous switching approach", Labour Economics, vol. 12, N 4, Amsterdam, Elsevier.

Hachen, D.S. Jr. (1992), "Industrial characteristics and job mobility rates", American Sociological Review, vol. 57, $\mathrm{N}^{\circ} 1$, Washington, D.C., American Sociological Association.

Holzer, H.J., J.I. Lane y L. Vilhuber (2003), "Escaping low earnings: the role of employer characteristics and changes", Discussion Paper, $\mathrm{N}^{\circ} 1269-03$, Institute of Research on Poverty.

IPEA (Instituto de Investigación Económica Aplicada) (2010), “O Brasil em 4 décadas", Texto para Discussão, $\mathrm{N}^{\circ} 1500$, Brasilia.

Jannuzzi, P.M. (2004), “As ocupações brasileiras segundo а сво 2002: caracterização empírica com base no Censo 2000", Revista da ABET, vol. 4, $\mathrm{N}^{\circ}$ 2, Associação Brasileira de Estudos do Trabalho (ABET).

Kambourov, G. e I. Manovskii (2004), "Rising occupational and industry mobility in the United States: 1968-1993", IZA Discussion Paper, $\mathrm{N}^{\circ} 1110$, Bonn, Institute for the Study of Labor (IZA).

Koenker, R. (2000), Quantile Regression, Illinois, Universidad de Illinois.

Koenker, R. y G. Basset (1978), "Regression quantiles", Econometrica, vol. 46, $\mathrm{N}^{\circ}$ 1, Washington, D.C., The Econometric Society.

Maciel, M.C., A.C. Campelo y M.C.F. Raposo (2001), "A dinâmica das mudanças na distribuição salarial e no retorno em educação para mulheres: uma aplicação de regressão quantílica", Anais do XXIX Encontro Nacional de Economia, Río de Janeiro, Asociación Nacional de Centros de Posgrado en Economía (ANPEC).

Maltseva, I. (2005), "Gender differences in occupational mobility and segregation at the labor market: the case of Russian economy", EERC Working Paper Series, N $^{\circ}$ 05/11, EERC Research Network.
Matos, R.S.E. y A.F. Machado (2006), "Diferencial de rendimento por cor e sexo no Brasil (1987-2001)”, Econômica, vol. $8 \mathrm{~N}^{\circ} 1$, Río de Janeiro, Universidad Federal Fluminense.

McLaughlin, K.J. (1991), "A theory of quits and layoffs with efficient turnover", Journal of Political Economy, vol. 99, $\mathrm{N}^{\circ}$ 1, Chicago, University of Chicago Press.

Mello, R.F. y D.D. Santos (2009), "Aceleração educacional e a queda recente da informalidade", Boletim de Mercado de Trabalho, $\mathrm{N}^{\circ}$ 39, Río de Janeiro, Instituto de Investigación Económica Aplicada (IPEA).

Menezes-Filho, N. (2004), "The costs of displacement in Brazil", XXVI Encontro Brasileiro de Ecomometria, João Pessoa, Sociedad Brasileña de Econometría.

Mincer, J. y B. Jovanovic (1979), "Labor mobility and wages", NBER Working Paper, $\mathrm{N}^{\circ}$ 357, Cambridge, Massachussets, National Bureau of Economic Research.

Monsueto, S.E. (2008), "Movilidad ocupacional e impactos sobre la renta en Brasil", tesis de doctorado, Madrid, Universidad Autónoma de Madrid (UAM).

Neri, M. y otros (1997), "Aspectos dinâmicos do desemprego e da posição na ocupação", Encontro Nacional de Estudos do Trabalho, Río de Janeiro, Associação Brasileira de Estudos do Trabalho (ABET).

Oliveira, A.M.H.C. (2003), "A segregação ocupacional por gênero e seus efeitos sobre os salários no Brasil", Mercado de trabalho: uma análise a partir das pesquisas domiciliares no Brasil, S. Wajnman y A.F. Machado (orgs.), Belo Horizonte, Editora UFMG.

(1998), "Indicadores da segregação ocupacional por sexo no Brasil", Anais do XI Encontro Nacional de Estudos Populacionais, Asociación Brasileña de Estudios Poblacionales (ABEP)

Oliveira, A.M.H.C. y A.F. Machado (2000), "Mobilidade ocupacional e rendimentos no Brasil metropolitano: 1991/96", Pesquisa e Planejamento Econômico, vol. 30, $\mathrm{N}^{\circ}$ 1, Instituto de Investigación Económica Aplicada (IPEA).

Oliveira, A.M.H.C. y P.M. Ribeiro (1998), "Diferenciais ocupacionais por raça e gênero no mercado de trabalho metropolitano no Brasil", XI Encontro Nacional de Estudos Populacionais, Asociación Brasileña de Estudios Poblacionales (ABEP).

Orellano, V. y P. Picchetti (2001), "A bi-variate probit analisys of job turnover in Brazil", XXIII Encontro Brasileiro de Econometria, Salvador, Sociedad Brasileña de Econometría.

Paci, P. y P. Serneels (ed.) (2007), Employment and Shared Growth: Rethinking the Role of Labor Mobility for Development, Washington, D.C., Banco Mundial.

Parrado, E., A. Caner y E. Wolff (2007), "Occupational and industrial mobility in the United States", Labour Economics, vol. 14, $\mathrm{N}^{\circ} 3$, Amsterdam, Elsevier.

Parrado, E. y E. Wolff (1999), "Occupational and industry mobility in the United States, 1969-1992", Working Papers, N 9920, Nueva York, C.V. Starr Center for Applied Economics, Universidad de Nueva York.

Pianto, M.T. y D.M. Pianto (2002), "Informal employment in Brazil - a choice at the top and segmentation at the bottom: a quantile regression approach", Textos para Discussão, $\mathrm{N}^{\circ} 236$, Brasilia, Departamento de Economía/Universidad de Brasilia.

Pinto, A. y M. Neri (2000), "Mobilidade ocupacional e raça: origens, destinos e riscos dos afro-brasileiros", Ensaios Econômicos, №392, Río de Janeiro, Fundación Getulio Vargas.

Ribas, R.P. y S.D.S. Soares (2008), "Sobre o painel da pesquisa mensal de emprego (PME) do IBGE", Texto para Discussão, $\mathrm{N}^{\circ}$ 1348, Río de Janeiro, Instituto de Investigación Económica Aplicada (IPEA)

Rocha, S. (2000), "Pobreza e desigualdade no Brasil: o esgotamento dos efeitos distributivos do Plano Real", Texto para Discussão, $\mathrm{N}^{\circ} 721$, Río de Janeiro, Instituto de Investigación Económica Aplicada (IPEA) 
Ruesga, S.B., J.S. Bichara y S.E. Monsueto (2009), "Diferencial de gênero e efeitos da mobilidade socioeconômica", XXXVII Encontro Nacional de Economia, Río de Janeiro, Asociación Nacional de Centros de Posgrado en Economía (ANPEC).

Sampaio, A.V. (2009), "Estimação da equação de salário para o Brasil, o Paraná e o Rio Grande do Sul em 2007 - uma abordagem quantílica", Indicadores Econômicos FEE, vol. 37, $\mathrm{N}^{\circ}$ 2, Porto Alegre, Fundação de Economia e Estatística.
Soares, C. y S. Oliveira (2004), "Gênero, estrutura ocupacional e diferenciais de rendimento", Econômica, vol. 6, N 1, Río de Janeiro, Universidad Federal Fluminense.

Souza, E.C. y J.E. Lima (2011), "Condição de ocupação e informalidade no mercado de trabalho brasileiro em 2007", Análise Econômica, vol. 29, № 56, Porto Alegre, Universidad Federal de Rio Grande do Sul (UFRGS).

Zimmermann, K.F. (1998), "German job mobility and wages", IZA Discussion Papers, $\mathrm{N}^{\circ} 4$, Bonn, Institute for the Study of Labor. 